\title{
Antiangiogenesis immunotherapy induces epitope spreading to Her-2/neu resulting in breast tumor immunoediting
}

This article was published in the following Dove Press journal:

Breast Cancer:Targets and Therapy

3 October 2009

Number of times this article has been viewed

\author{
Matthew M Seavey \\ Yvonne Paterson \\ Department of Microbiology, \\ University of Pennsylvania School \\ of Medicine, Philadelphia, PA, USA
}

Correspondence: Yvonne Paterson Department of Microbiology, 323 Johnson Pavilion, 36th street and Hamilton Walk, University of Pennsylvania School of Medicine, Philadelphia, PA 19104-6076, USA

Email yvonne@mail.med.upenn.edu
Abstract: Targeting tumors using cancer vaccine therapeutics has several advantages including the induction of long-term immunity, prime boost strategies for additional treatments and reduced side effects compared to conventional chemotherapeutics. However, one problem in targeting tumor antigens directly is that this can lead to antigen loss or immunoediting. We hypothesized that directing the immune response to a normal cell type required for tumor growth and survival could provide a more stable immunotherapeutic target. We thus examined the ability of an antiangiogenesis, Listeria monocytogenes (Lm)-based vector to deliver extracellular and intracellular fragments of the mouse vascular endothelial growth factor receptor-2/Flk-1 molecule, Lm-LLO-Flk-E1, and Lm-LLO-Flk-11 respectively, in an autochthonous model for Her- $2 / \mathrm{neu}^{+}$breast cancer. We found that these vaccines could cause epitope spreading to the endogenous tumor protein Her-2/neu and significantly delay tumor onset. However, tumors that grew out overtime accumulated mutations in the Her- $2 /$ neu molecule near or within cytotoxic T lymphocytes epitopes. We show here for the first time how an antiangiogenesis immunotherapy can be used to delay the onset of a spontaneous tumor through epitope spreading and determine a possible mechanism of how immunoediting of an endogenous tumor protein can allow for tumor escape and outgrowth in an autochthonous mouse model for Her-2/neu ${ }^{+}$breast cancer.

Keywords: Listeria, Her-2/neu, immunotherapy, antiangiogenesis, immunoediting

\section{Introduction}

Cancer immunotherapy has expanded in the past several years to include therapies utilizing whole cell vaccines, modified antigen-presenting cells, adoptive cell and antibody therapy, live and dead viral vectors, live bacteria and peptide, protein, and DNA vaccines. ${ }^{1-3}$ The benefit of using the immune system to target tumors is that toxicity to self-tissues is mitigated. However, many tumor antigens are modified and/or overexpressed self-proteins. Overcoming tolerance to self-proteins requires breaking peripheral tolerance. Thus, the inflammatory cascade induced by a cancer vaccine must be great enough to break tolerance to the protein targeted.

Directly targeting tumors using the live bacterial vector, Listeria monocytogenes $(\mathrm{Lm})$, is sufficient to break tolerance to several known tumor antigens ${ }^{4-7}$ and repeatedly has been shown to induce tumor regression or stasis in both mouse and humans. ${ }^{3}$ Its efficacy is dependent on the actions of antitumor cytotoxic T lymphocytes (CTL). ${ }^{5,8,9}$ The difficulty with immunotherapy is that the recognition of tumor proteins by CTLs is entirely dependent on the recognition of a unique peptide- major histocompatibility complex (MHC-I) that requires intimate interaction with key 
domains of the $\mathrm{T}$ cell receptor (TCR). Mutation of any of these components can result in the failure to recognize and kill a growing tumor. Immunological pressure can lead to immunoediting of key amino acids required for CTL recognition. ${ }^{10-12}$ Targeting tumors directly using anti-Her-2/neu Lm vaccines can delay spontaneous tumor growth, especially when the region targeted is in a key kinase domain responsible for cell signaling and tumor outgrowth. ${ }^{11}$ The problem with targeting tumors directly by immunizing with a self-tumor protein is that not only can immunoediting occur, but also the immediate tumor microenvironment may harbor many immunosuppressive factors and mechanisms that allow for easy escape.

We have devised strategies to target the tumor vasculature using Listeria technology. Cloning fragments of the mouse vascular endothelial growth factor receptor-2 (VEGFR2) gene, Flk-1, we were able to cause the active regression of established breast tumors in vivo and subsequently delay tumor growth in the lungs of protected mice. ${ }^{13}$ Here we describe a mechanism by which spontaneous tumor outgrowth is significantly delayed when tumor angiogenesis is targeted. This demonstrates that the previously reported epitope spreading observed with these vaccines ${ }^{13}$ can overcome tolerance to an endogenous tumor-associated protein and both delay the outgrowth of autochthonous tumors and induce mutations in key domains of a tumor protein responsible for growth and propagation.

Nearly 200,000 women are diagnosed with breast cancer each year in the US alone, which correlates to an incidence of one in eight women, and about 40,000 women die from breast cancer per year. The EGFR-like receptor, Her-2/neu, is over-expressed in about $15 \%-40 \%$ of all breast cancers and has been shown to be expressed on carcinomas of the pancreas, colon, and ovaries. ${ }^{14} \mathrm{Her}-2 /$ neu overexpression is usually associated with poor prognosis, and Her-2/neu-expressing tumor cells are often chemoresistant and most likely to metastasize and colonize the bone and brain in terminal patients. ${ }^{15,16}$ Here we used a transgenic animal model for human breast cancer. The overexpression of rat Her-2/neu under the control of the MMTV promoter induces spontaneous outgrowth of Her-2/neu positive tumors in the mammary fat pad of aging mice. We show for the first time that targeting the vasculature using an Lm-based vaccine can significantly delay the outgrowth of spontaneous tumors, induce epitope spreading to an autochthonous tumor antigen, and drive immunoediting of a key tumor protein responsible for breast tumor growth.

\section{Materials and methods \\ Mice}

The FVB/N Her-2/neu transgenic mice were housed and bred at the animal core facility at the University of Pennsylvania. Mice were six to eight weeks old when used at the start of the experiments, which were performed in accordance with regulations by the Institutional Animal Care and Use Committee of the University of Pennsylvania.

\section{Peptides and antibodies}

Rat anti-mouse interferon- $\gamma$ (IFN $\gamma$ ) (clone AN18) was purchased from MABTECH (Mariemont, OH). Rat anti-mouse IFN $\gamma$ (clone XMG1.2) was purchased from eBioscience (San Diego, CA). All peptides were purchased from EZBiolabs (Westfield, IN). Peptides used in this paper were as follows: $\mathrm{Neu}_{1022-1041}=$ WT 1022-1041 (VPQQGFFSPDPTPGTGSTAH), $\mathrm{Neu}_{1026-1036}=\mathrm{WT}$ 1026-1036 (GFFSPDPTPGT), $\mathrm{Neu}_{1032-1041}=$ WT 1032-1041 (PTPGTGSTAH), $\mathrm{Neu}_{\mathrm{F} 1027 \mathrm{I}}=$ Mut F1027I (GIFSPDPTPGT), $\mathrm{Neu}_{\mathrm{G} 1035 \mathrm{~K}}=$ Mut G1035K (GFFSPDPTPKT), pHIV Gag (AMQMLKETI), ${ }^{17}$ Flk-11 $1_{906-915}$ (PGGPLMVIV). ${ }^{18}$

\section{Listeria vaccine strains and DNA vaccines} Strains used were Lm-LLO-Flk-E1 and Lm-LLO-Flk-11. ${ }^{13}$ The strain Lm-LLO-NY-ESO-1 was used as a third party control vaccine for antigen specificity. ${ }^{19}$ Bacteria were selected on brain heart infusion (BHI; Difco) plates supplemented with $34 \mu \mathrm{g} / \mathrm{ml}$ of chloramphenicol and $250 \mu \mathrm{g} / \mathrm{ml}$ of streptomycin, then grown in liquid culture and frozen in $1 \mathrm{ml}$ aliquots at $-80{ }^{\circ} \mathrm{C}$. For injection, the vaccines were washed twice with sterile phosphate-buffered saline (PBS) before administration. DNA vaccines used in this paper were used to induce anti-Her-2/neu responses in wild-type FVB/N mice. DNA vaccines were as follows: pcDNA3.1-LLO-Neu, pcDNA3.1-MIP-1 $\alpha$, and pcDNA3.1-GM-CSF (gifts from Dr. David Weiner, Department of Pathology, University of Pennsylvania, Philadelphia, PA). Construction and use are described elsewhere. ${ }^{7,20}$

\section{Autochthonous tumor protection}

To test the ability of anti-Flk-1 Listeria vaccines to impact on spontaneously arising tumors we used the FVB/N rat Her-2/neu transgenic female mouse which overexpresses the rat Her-2/neu molecule and spontaneously develops mammary tumors around 21-35 weeks of age. For these long-term protection studies, we immunized female mice $(\mathrm{N}=15)$ a total of six times starting at six weeks of age and immunizing intraperitoneal every three weeks until 21 weeks of age. 
Vaccines Lm-LLO-Flk-E1, Lm-LLO-Flk-11, or control Lm-LLO-NY-ESO- 1 was injected at $1 \times 10^{8}$ colony-forming units (CFUs) suspended in PBS. Tumor burden was followed on a weekly basis. Once tumors were beyond $10 \mathrm{~mm}$ in size animals were sacrificed and tumors were removed for analysis. Statistical analysis of differences in autochthonous tumor growth was done using the Kaplan-Meier log-rank test using GraphPad Prism software (GraphPad, La Jolla, CA), comparing the time of onset of tumor growth between each vaccine group and control groups.

\section{Analysis and mapping of mutations}

Tumors were excised fresh and placed into RNAlater solution (Ambion, Austin, TX), stored at $4{ }^{\circ} \mathrm{C}$ for less than two weeks. We extracted mRNA from stored tumors using a Qiagen RNeasy kit (Qiagen, Valencia, CA). We then generated cDNA via PCR using an Applied Biosystems high capacity cDNA reverse transcription kit (Applied Biosystems, Foster City, CA). PCR reactions were performed using PureTaq RTG PCR Beads (GE Healthcare, Piscataway, NJ). Individual PCR samples were further divided to allow sequencing of each individual fragment of Her-2/neu in stretches of 500-800 bp each (EC1 [AA 20-326], EC2 [AA 303-501], EC3 [AA 479-655], IC1 [AA 690-1081], IC2 [AA 10201260]) as was described elsewhere. ${ }^{11}$ Primers for these reactions are as follows: EC1 (F): 5'-AGGGCTGTCAGGTAGTGC-3', EC1 (R): 5'-TGACCTCTTGGTTATTCG-3', EC2 (F): 5'-ACCTGCCCCTACAACTAC-3', EC2 (R): 5'-GACGCCCTCTACAGTTGC-3',EC3(F): 5'-GTGGATTGGCTCTGATTC-3', EC3 (R): 5'-TGAGTTACAGACCAAGCC-3', IC1 (F): 5'-CAAACGAAGGAGACAGAAG-3', IC1 (R): 5'-CACCATCAAACACATCGG-3', IC2 (F): 5'-CACTGCTGGAAGATGATG-3', IC2 (R): 5'-TTTGTGGCGATGGAGACC-3'. Sequencing was done by the Children's Hospital of Philadelphia (CHOP) Sequencing Facility and then analyzed using 4Peaks software (v. 1.7.2; Griekspoor and Groothuis, http://mekentosj. com/4peaks/). Mutations that did not occur in four or more individual PCR and sequencing reactions were discarded as PCR-induced mutations. Molecular modeling was done using MacPyMol software (DeLano Scientific, Palo Alto, CA).

\section{Cell lines}

Cell culture media and supplements were purchased from Gibco (Invitrogen, Carlsbad, CA). NT-2 cells were maintained in complete RPMI 1640 supplemented with $10 \%$ fetal calf serum (FCS), $2 \mathrm{mM}$ L-glutamine, $100 \mu \mathrm{M}$ nonessential amino acids, $100 \mathrm{U} / \mathrm{ml}$ penicillin-G and $100 \mu \mathrm{g} / \mathrm{ml}$ streptomycin. All cell cultures were kept at $37^{\circ} \mathrm{C}$ and $5 \% \mathrm{CO}_{2}$.

\section{ELISpot assays}

Secretion of IFN- $\gamma$ by mouse splenocytes in response to peptide stimulation was tested by enzyme-linked immunospot (Elispot) assay as previously described. ${ }^{21}$ Briefly, isolated splenocytes were plated at $5 \times 10^{6}$ cells per well or titrated across a 96-well plate coated with $7 \mu \mathrm{g} / \mathrm{ml}$ of rat anti-mouse IFN- $\gamma$ antibody (clone AN18, MABTECH), in the presence of $5 \mu \mathrm{g} / \mathrm{ml}$ peptide and $5 \mathrm{U} / \mathrm{ml}$ of IL-2. Secondary, biotinylated, anti-IFN $\gamma$ antibody (clone XMG1.2, eBioscience) was added to each well at a final concentration of $2 \mu \mathrm{g} / \mathrm{ml}$. After overnight incubation at $37{ }^{\circ} \mathrm{C}$, plates were developed for one hour at room temperature with Streptavidin-horseradish peroxidase (1:1000 dilution) followed by substrate TMB (ABC kit, Vector laboratories, Burlingame, CA). Spots were counted using the Immunospot CTL. scanner and counting software (CTL, Cleveland, $\mathrm{OH}$ ).

\section{Statistical analysis and methods of quantitation}

Data were analyzed using the nonparametric Mann-Whitney test. The log-rank chi-squared test was used for all survival data. All statistical analysis was done with Prism software, (v. 4.0a; GraphPad). Statistical significance was based on a value of $p \leq 0.05$. In all nontransgenic studies we included at least eight mice per group. For all FVB/N rat Her-2/neu transgenic studies we used at least 15 mice per group.

\section{Results \\ Delay in indolent breast tumor growth following anti-Flk-I vaccination}

Previously we showed that vaccination of tumor-bearing mice with anti-Flk-1 Lm vaccines could lead to epitope spreading to the tumor-associated protein Her-2/neu, and that this secondary antitumor response was responsible for the observed regression in vivo. ${ }^{13}$ When spontaneous models for Her-2/neu ${ }^{+}$breast cancer were immunized, we observed a highly significant delay in tumor outgrowth when vaccinated against the extracellular (Flk-E1) and intracellular (Flk-11) portions of mouse VEGFR2/Flk-1 (Figure 1, $P<0.0001$ ). This delay was extended about 15 weeks beyond that of the control-treated mice. However, tumor outgrowth was observed starting around 35 weeks of age. Also, tumor growth was not restricted to a single mammary gland (ie, many different gland regions were involved) and observed tumor growth did not appear to differ between controls and treated mice (observations not shown). Since previous work targeting the Her-2/neu protein suggested that 


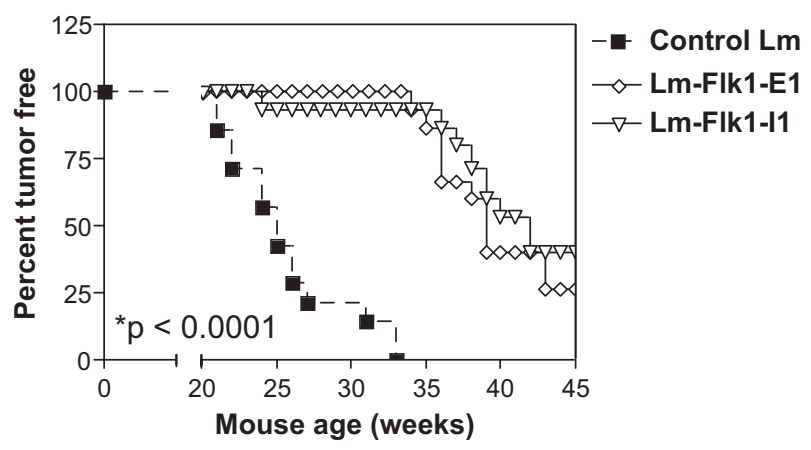

Figure I Tumor delay after treatment with anti-VEGFR2/Flk-I vaccines in an autochthonous model for Her-2/neu breast cancer. Six week old mice expressing a transgene for rat Her-2/neu were vaccinated with a I $\times 10^{8} \mathrm{CFU}$ of Lm-LLO-FIk-EI, Lm-LLO-Flk-I I, or a control Lm i.p. for a total of six sequential treatments. Each vaccine was given every three weeks, from six weeks and continuing to 21 weeks of age at which time mice were observed on a weekly basis for the presence of mammary tumors. Mice were followed out beyond 55 weeks. $N=15$ mice per group. At 55 weeks, $0 / 15$ mice were tumor negative for the control Lm group, $3 / 15$ were negative for both the Flk-EI and Flk-II groups. Rank-log test comparing the Flk-EI and Flk-I I groups to the control Lm groups give a highly significant value of $* P<0.0001$.

Abbreviation: Lm, Listeria monocytogenes.

tumor outgrowth was due to mutations that had accumulated in the Her-2/neu protein allowing tumor escape, ${ }^{11}$ we decided to test whether the same phenomenon was occurring under immune pressure from these antiangiogenesis vaccines.

\section{Specific mutations accumulate in Her-2/neu following Lm-LLO-Flk-I vaccination}

Mice around 40 weeks of age were removed from the study. A single, independent tumor from each mouse was removed and analyzed for Her-2/neu mutations (Table 1). Tumor mRNA was extracted and reverse transcribed, PCR amplified and sequenced for five different regions of the Her-2/neu molecule as previously described. ${ }^{11}$ These five rat Her-2/neu regions were chosen based on previous regions that we used to vaccinate mice against the rat Her-2/neu molecule in our original study. ${ }^{11}$ Three of these regions covered the extracellular domain (EC1, EC2, EC3) and two covered the intracellular domain of HER-2/neu (IC1, IC2). Each region corresponds to an overlapping Her-2/neu amino acid sequence: EC1 (AA 20-326), EC2 (AA 303-501), EC3 (AA 479-655), IC1 (AA 690-1081), or IC2 (AA 1020-1260). Mutations that occurred in four or more PCR sequencing reactions using both forward and reverse primers were considered to be real mutations and not PCR-induced mutations. Three or less mutations of a single amino acid were dropped from further analysis. Our selection of four or more mutated residues acts as an analysis cut off and was pursued based on a previously published report using a similar strategy for identifying now confirmed epitopes within unmapped regions of rat Her-2/neu. ${ }^{11}$ Bolded residues correspond to amino acids that also undergo mutation when the vaccination is directed at Her-2/neu. ${ }^{11}$ These are the residues most likely to mutate under immunological pressure either via direct vaccination or indirectly via epitope spreading.

At least three mice from each group were tested including the control tumors. We clearly see more mutations occurring in Her-2/neu from tumors from treated versus untreated control mice (Table 1). Only two residues were mutated in tumors from control vaccinated mice and neither of these occurred in known CTL epitopes. The Flk-E1 vaccinated mice show more mutations in the extracellular fragment than the intracellular fragment, however, Flk-11 show mutations in both fragments. Also, the Flk-11 mutations seem to occur at a higher frequency in the region between residues 1026 and 1051 and several mutations overlap with mutations previously identified from Her-2/neu directed vaccinations. ${ }^{11} \mathrm{We}$ do not see any correlation between frame shift, substitutions, deleted residues, and vaccination. It is possible that certain regions of the Her-2/neu molecule are susceptible to mutation or sensitive to selective pressure, such that certain mutations do not impact on signaling and tumor growth but do provide a selective advantage for survival.

When we summarize all individual mutations and overlay the mutated residues on the full amino acid sequence for the rat Her-2/neu gene product (Figure 2), we notice the same pattern: 1) More mutations occur in tumors from treated versus untreated mice (Figure 2A vs Figure 2B-C, highlighted residues), 2) Tumors from mice vaccinated with Flk-E1 show mutations mainly in the extracellular fragment (Figure 2B), whereas 3) those from mice treated with Flk-11 show mutations in both the extracellular and intracellular fragments (Figure 2C). In addition, we did not observe any mutations within the Her-2/neu kinase domain (italics) nor any in the adenosine triphosphate (ATP)-binding sequence (VLGSGAFGTV) within this domain. Both the kinase domain and ATP-binding domain are conserved amino acid regions necessary for proper receptor signaling. ${ }^{22,23}$ However, several of the mutations are grouped and seem to occur in 'hot spots' or areas that are more likely to become mutated under immunological pressure. Further investigating these 'hot spots' we see two regions of interest, one occurring in the Flk-E1-treated mice, in the previously identified CTL epitope: LFRNPHQALL ${ }^{24}$ and another 'hot spot' corresponding to an unmapped region that may contain new CTL epitopes: QQGFFSPDPTPGTGSTA. 


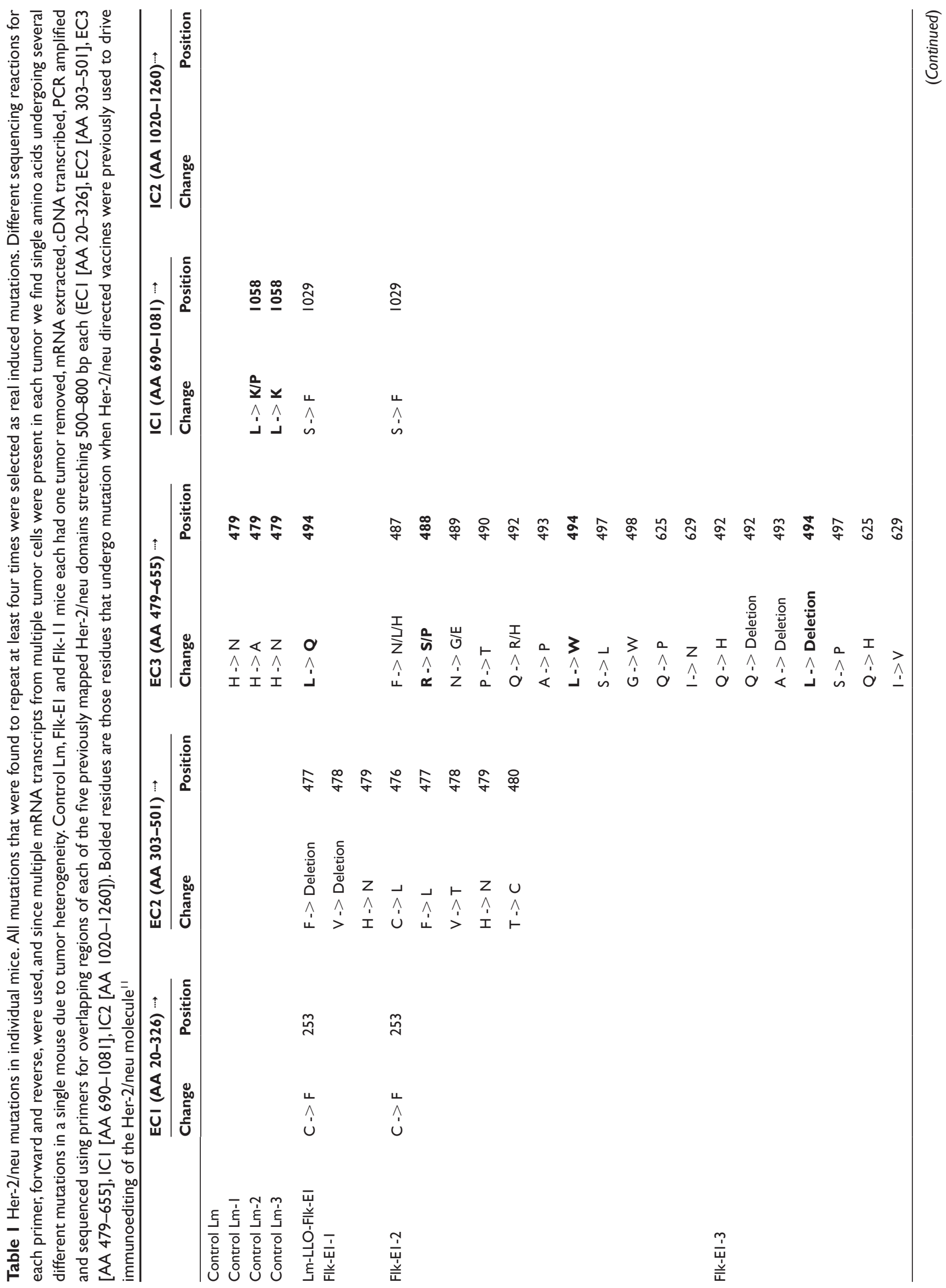




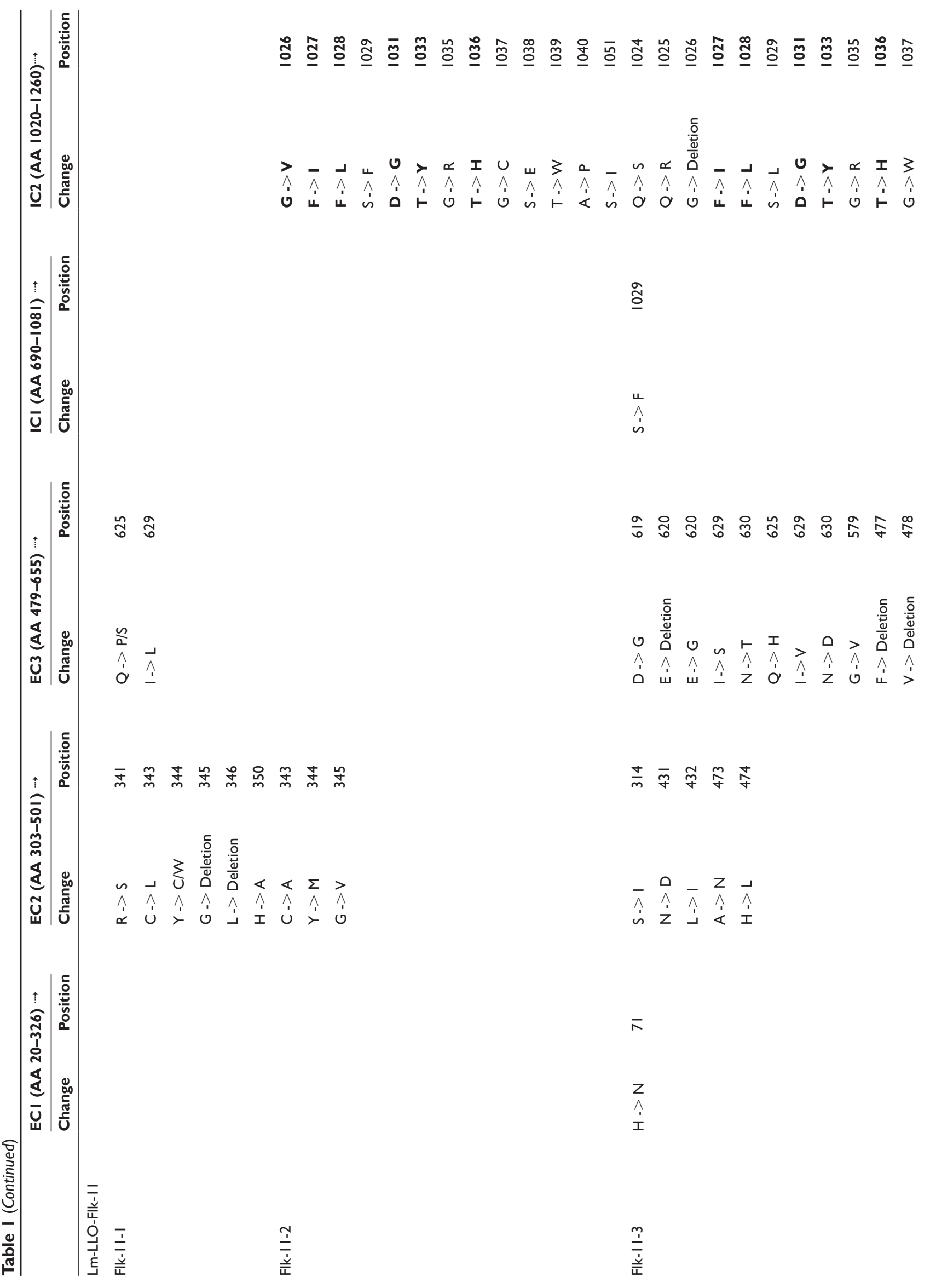




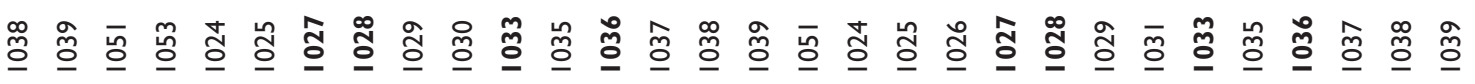

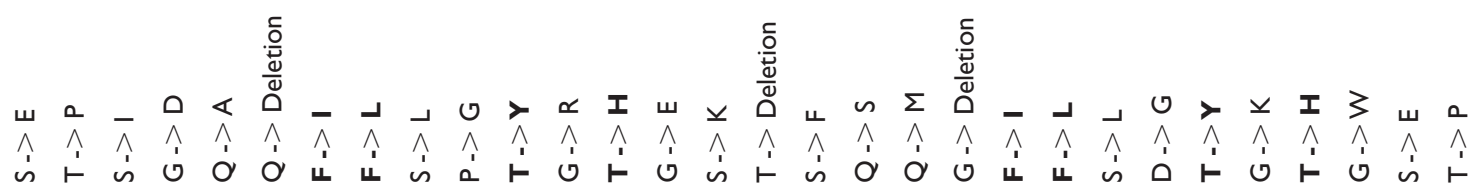

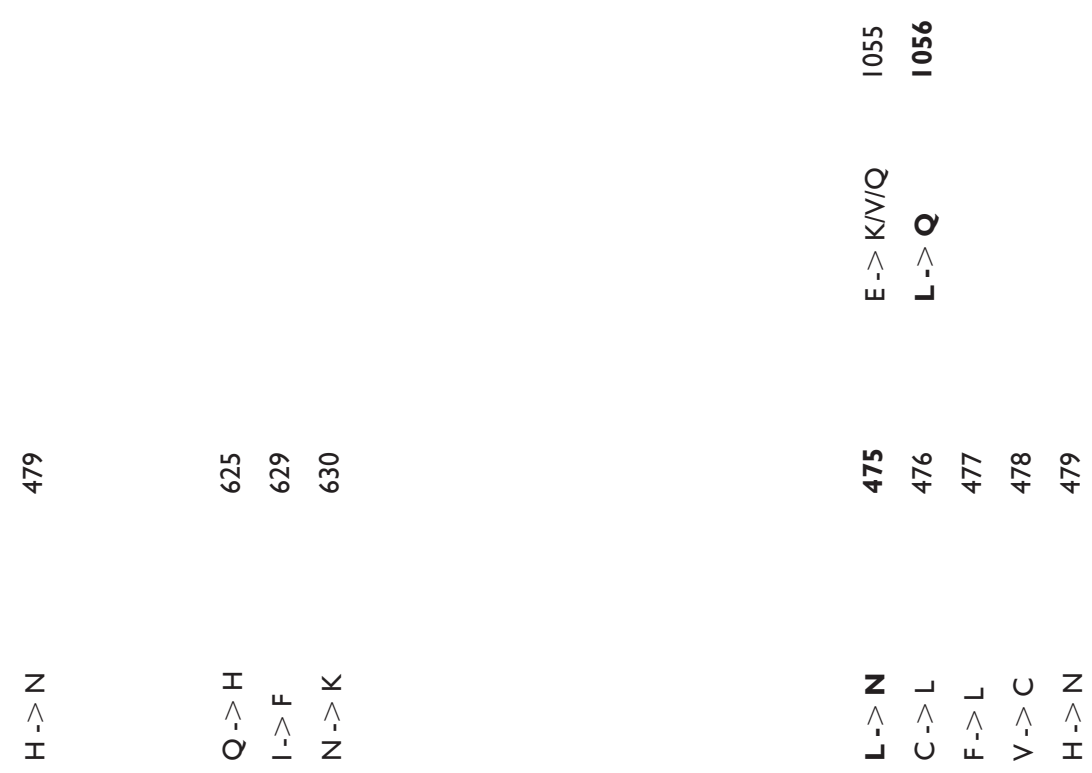

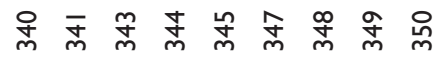

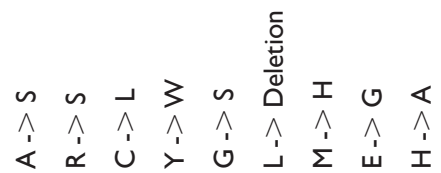

กิ โ

$\begin{array}{ll}> & \sim \\ \hat{u} & \hat{o}\end{array}$

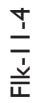

$\frac{\stackrel{1}{\overline{1}}}{\frac{\dot{y}}{\bar{H}}}$ 


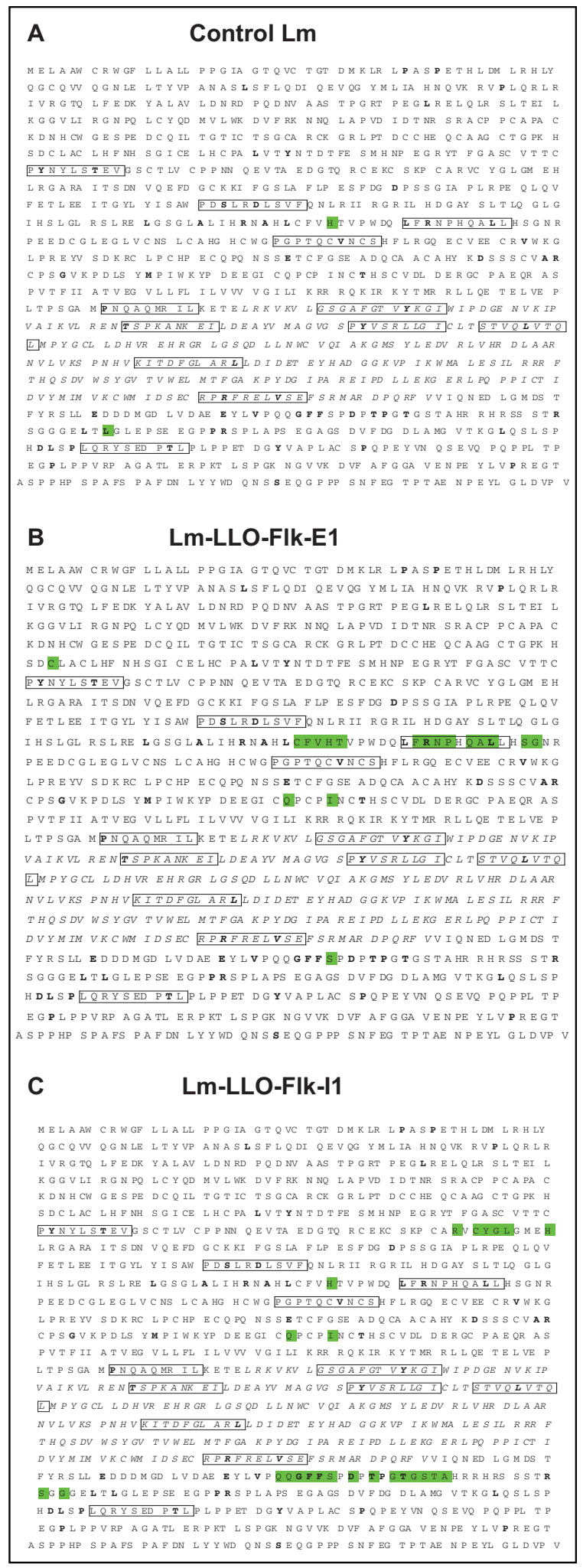

Figure 2 Summary of individual mutations overlaid on the wild-type Her-2/neu amino acid sequence. Summary of all mutations were overlaid onto the full wild-type sequence of rat Her-2/neu. Bolded amino acids are residues that undergo mutation with the Her-2/neu Lm vaccine, " residues in dark shaded highlight are residues that undergo mutation after A) control B) Flk-EI, or C) Flk-I I Lm vaccination. Selected italic sequence denotes the kinase domain. Boxed sequences are previously identified CTL epitopes. ${ }^{24}$ Sequence overlays contain compressed data from Table I. Abbreviation: CTL, cytotoxic T lymphocytes.

\section{Her-2/neu mutations from mice vaccinated with the kinase domain of VEGFR2 fall within two unidentified CTL epitopes that when mutated abrogate $\mathrm{CD}^{+} \mathrm{T}$ cell responses ex vivo}

Several regions are mutated when mice are treated with Flk-11 vaccine (Figure 2C), so we decided to test if any of these regions could contain possible CTL epitopes. We tested synthesized 20mers from various 'hot spot' regions using an IFN- $\gamma$ Elispot after vaccination of wild-type mice with Her-2/neu DNA vaccines (Figure 3). The different Her-2/neu 20mer regions are as follows: 1022-1041, VPQQGFFSPDPTPGTGSTAH; 621-640, EGICQPCPINCTHSCVDLDE; 619-638, DEEGICQPCPINCTHSCVDL; 606-625, KPDLSYMPIWKYPDEEGICQ; 486-505, LFRNPHQALLHSGNRPEED; 471-490, RNAHLCFVHTVPWDQLFRNP; 456-475, LRSLRELGSGLALIHRHAH. We determined that all regions contained epitopes, but that the 1022-1041 region contained epitopes with the greatest potential to induce $\mathrm{CD} 8^{+} \mathrm{T}$ cell restricted responses. Upon closer investigation of this region we noticed a stretch of amino acids that accumulated several mutations when anti-Her-2/neu Lm vaccines were previously used in this model. ${ }^{11}$ The sequence VPQQGFFSPDPTPGTGSTAH, when analyzed using the epitope predictor programs RANKpep (http://bio. dfci.harvard.edu/RANKPEP/) and SYFPEITHI (http://www. syfpeithi.de/) resulted in the generation of two potential epitopes each for a different MHC-1 gene product, $\mathrm{H} 2 \mathrm{~K}$ and $\mathrm{H} 2 \mathrm{D}$. We thus synthesized the putative $\mathrm{H} 2 \mathrm{~K}^{\mathrm{d} / \mathrm{q}}$ binding peptide GFFSPDPTPGT and the $\mathrm{H} 2 \mathrm{D}^{\mathrm{d} / q}$ binding peptide TPGTGSTAH. After careful consideration of all the various mutations and binding affinities for both $\mathrm{H} 2 \mathrm{~K}$ and $\mathrm{H} 2 \mathrm{D}$ molecules, we decided to synthesize two mutant peptides, one changing a phenylalanine to an isoleucine (F1027I) and another changing a glycine to a lysine (G1035K), both mutations occur naturally and were picked from Table 1 as representative mutations (Figure 4A).

To test if epitope spreading to Her-2/neu had occurred in the transgenic mice, and if responses to these Her-2/neu epitopes were abrogated after mutation of certain residues, we proceeded to test the remaining mice that had not yet developed tumors, and were still considered protected from disease, shown in Figure 1. Saline-treated control mice were from age-matched females that had already formed spontaneous tumors similar to the control Lm-treated mice in Figure 1. Splenocytes from each animal were tested for the ability to recognize $\mathrm{CD} 8^{+} \mathrm{T}$ cell epitopes from wild-type 


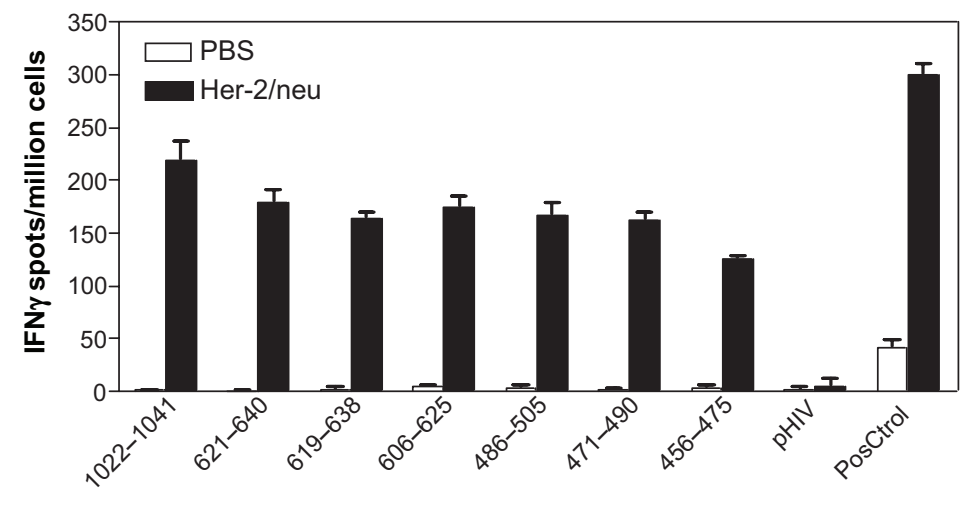

Rat Her-2/neu 20 mer peptides

1022-1041 = VPQQGFFSP DPIP GTGSTAH

621-640 = EGICQPCPINCTHSCVDLDE

619-638 = DEEGICQPCPINCTHSCVDL

606-625 = KPDLSYMPIWKYPDEEGIC $\underline{Q}$

486-505 = LFRNPHQALLHSGNRPEED

471-490 = RNAHLCFVHTVPWDQ LFRNP

456-475 = LRSLRE LGSGLALIHRNAH

$A A=$ Her-2/neu sequence

AA $=$ Mutated with Neu vaccines

$\mathrm{AA}=$ Mutated with Flk vaccines

$A A=$ Neu CTL epitope

Figure 3 Mutated regions in Her-2/neu contain CTL epitopes. FVB/N wild-type mice were either immunized with Her-2/neu DNA vaccines or saline/PBS alone three sequential times each spaced one week. Splenocytes were tested ex vivo for their ability to recognize 20 mer peptides that overlapped mutated regions. Graph shows number of IFN $\gamma$ spots per million splenocytes rechallenged using 20 mer peptides. Bolded residues are those amino acids that have undergone mutation using the Her-2/neu vaccines, italics are previously identified CTL epitopes, ${ }^{24}$ and underlined residues are those amino acids that undergo mutation using Flk-I vaccines. PMA/lonomycin were used as a positive control (PosCtrol), pHIV Gag (AMQMLKETI) peptides was used as a third party control. Media alone well were subtracted from all groups as a background control. Graph shows mean \pm SEM.

Abbreviations: CTL, cytotoxic T lymphocytes; PBS, phosphate-buffered saline; SEM, standard error of mean.

regions $\mathrm{Neu}_{1026-1036}$ : GFFSPDPTPGT and $\mathrm{Neu}_{1032-1041}$ : PTPGTGSTAH (Figure 4B). Both peptides stimulated a response ex vivo although $\mathrm{Neu}_{1032-1041}$ was better than $\mathrm{Neu}_{1026-1036}$. However, when mutations were introduced at $\mathrm{Neu}_{1027}$ and $\mathrm{Neu}_{1035}$ ex vivo responses were eliminated, even in the saline-treated animals. which suggests that this epitope is responsible even for recognition of endogenous Her-2/neu without vaccination. These data provide evidence that protection against spontaneous Her-2/neu positive tumors can be achieved after vaccination against certain regions of the VEGFR2 receptor, accompanied by epitope spreading to Her-2/neu, but that immunoediting of key domains in molecules responsible for tumor progression may hinder the therapeutic application of this therapy.

\section{Discussion}

The difficulty with targeting tumors, in general, is that with time the instability of tumor cells, combined with rapid growth kinetics, eventually leads to sustainable mutations. With the pressure of therapeutic selection, mutations that allow tumor escape can eventually emerge. For small molecule and chemotherapeutics, depending on the target and broad reach of the compound, some tumors will require several mutations before growing out. However, the use of chemotherapeutics has numerous problems, especially with toxic side effects. The power of immunotherapy has always been that tumors could be targeted with minimal toxic side effects or damage to normal tissues, and would provide long-lasting immunity via the production of memory $\mathrm{T}$ and $\mathrm{B}$ cells. In addition, targeting the vasculature using antiangiogenesis immunotherapy may not only augment ongoing antitumor immunity, but it may also be combined with current standard of care for a synergistic effect. However, targeting processed proteins becomes a problem when tumor cells are able to mutate genes responsible for not only the targeted protein but also machinery required for the processing of these gene products, such as the proteosome and ubiquin pathway proteins. ${ }^{25-27} \mathrm{We}$ believe that mutations are occurring everywhere in the genome, but only those tumors grow that generate mutations that are beneficial for survival and select for tumor growth and thus escape. If these mutations do not hinder the normal functions of an essential protein or pathway, then this should result in a tumor mass weeks to months after several rounds of targeted vaccination. This is observed in this study using a long-term spontaneous model that requires 35 weeks before tumor onset. Constant pressure from multiple rounds of vaccination (six total) appears to have 
A

Mutant F1027I: G포 SPDPTPGT

Mutant G1035K: GFFSPDPTPKT

Wild-type: GFFSPDPTPGT PTPGTGSTAH

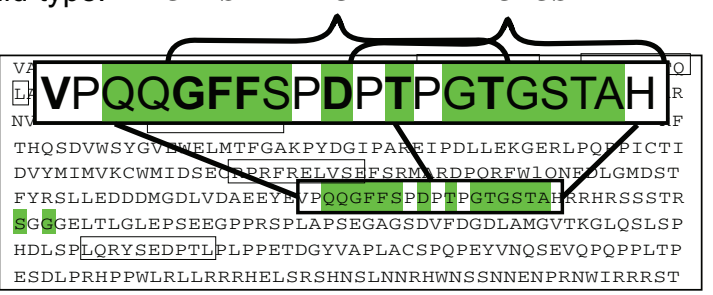

B

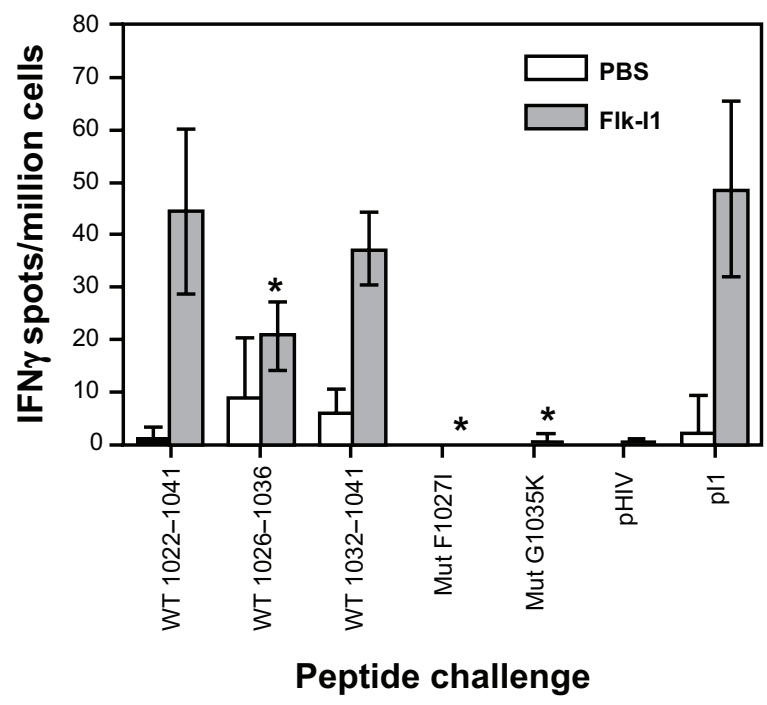

Figure 4 Epitope spreading to a previously unmapped Her-2/neu epitope occurs in response to FLK-I vaccination in transgenic mice and $\mathrm{T}$ cell recognition is abrogated by accumulated mutations in this epitope. A) Wild-type and mutant peptides were synthesized for each region of interest from the $\mathrm{Neu}_{1022-1041} 20$ mer peptide that showed the best response in re-challenge assays ex vivo and contained several mutations from both Her-2/neu and Flk-I vaccinations. Mutations included a FI027I and G $035 \mathrm{~K}$ residue substitution. This substitution was based on prediction mapping using RANKpep and data from Table I.B) Remaining mice that had not yet developed tumors, around week 55, were age-matched with saline-treated mice that had accumulated tumors and were analyzed for epitope spreading to this unmapped region and ability to recognize mutated versions that would most likely be expressed by the spontaneous tumors. Graph shows ex vivo IFN $\gamma$ Elispot responses after rechallenge of splenocytes with the full 20 mer $\mathrm{Neu}_{1022-1041}$ peptide, and both fragments, $\mathrm{Neu}_{1026-1036}$ and $\mathrm{Neu}_{1032-1041}$, also mutant peptides $\mathrm{Neu}_{\mathrm{F} 10271}$ and $\mathrm{Neu}_{\mathrm{G} 1035 \mathrm{~K}} \cdot \mathrm{PlI}=\mathrm{Flk} \mathrm{II} \mathrm{I}_{906-915}$ (PGGPLMVIV), was used as a positive control for anti-Flk-I I responses. Media alone well were subtracted from all groups as a background control. Graph shows mean \pm SEM; $* P<0.05$; Mann-Whitney nonparametric test.

Abbreviation: SEM, standard error of mean.

selected for tumors that can evade cells responsible for their recognition and demise, ie, CTL.

It is not surprising that mutations arise in protected animals, as this is likely to occur in any cancer model under selective pressure. In addition, we would expect more mutations to occur in the Her-2/neu molecule than in the originally targeted Flk-1 molecule as the tumor is a genetically unstable cell type compared to the endothelium. However, this does provide further evidence for the importance of epitope spreading to Her-2/neu as a major mechanism for the efficacy of the antiFlk-1 vaccines, as we have proposed previously. ${ }^{13}$

Indeed, when one examines the regions of Her-2/neu that have accumulated mutations after vaccination, some mutations clearly arise within previously known CTL epitopes. One particular 'hot spot' of mutational activity occurs within a previously identified epitope residues 486-495: LFRNPHQALL. ${ }^{24}$ This epitope was previously shown to contain three mutations, shown in bold, that are induced via direct anti-Her-2/neu immunization. ${ }^{11}$ Here, we find seven mutations induced by immunization with at least one of the Flk-1 vaccines that partially overlap with the previously identified mutations. This epitope was originally mapped to $\mathrm{H} 2 \mathrm{~K}^{q / d} \cdot{ }^{24} \mathrm{H} 2 \mathrm{~K}^{\mathrm{q} / \mathrm{d}}$ uses position $2(\mathrm{P} 2)$ and position 9 (P9) for peptide anchoring. ${ }^{28}$ The consensus for $\mathrm{H} 2 \mathrm{~K}^{\mathrm{q} / \mathrm{d}}$ is the presence of a tyrosine or phenylalanine at $\mathrm{P} 2$ and an isoleucine or leucine at the P9 position. ${ }^{28}$ For this selected $10 \mathrm{mer}$ both $\mathrm{P} 2$ and $\mathrm{P} 9$ anchor positions are mutated when the Flk-E1 vaccine is used, whereas the $\mathrm{P} 3$, probably a TCR recognition residue, and $\mathrm{P} 9$, an anchor position, are mutated when the Her-2/neu vaccines are used. Table 1 shows that in several instances multiple mutations occurred in a single mouse because the sequence analysis may represent the overlapped sequences of Her-2/neu of thousands of different tumor cell types. However, certain positions are mutated consistently several times to different amino acids (eg, Table 1, F487).

It is of interest that most of the Flk-1-induced mutations do not occur in conserved domains such as the kinase domain or ATP-binding domain (Figure 2) whereas transgenic mice vaccinated with a Listeria vaccine that specifically targeted that domain in Her-2/neu did develop tumors with several Her-2/neu-induced mutations in the kinase domain, all of which were found to be within CTL epitopes. ${ }^{11}$ It is possible that there is a threshold for mutagenicity of certain regions of the gene that becomes breached under different levels of immunological pressure. For instance, vaccines that directly target the tumor pose a greater selective pressure than vaccines that target the tumor indirectly via an epitopespreading mechanism. The frequency of anti-Her-2/neu CTLs generated via a spreading mechanism will be far fewer in number and, possibly, affinity than CTLs generated by vaccines expressing the target molecule. ${ }^{29}$ This is a possible explanation for the increased number of single mutations in the Her-2/neu-directed vaccinations versus the far fewer number of observed single mutations in the Flk-1-directed vaccinations. 
Immunoediting is very similar to antigenic drift seen for some virus infections ${ }^{30}$ and parasitic infections. ${ }^{31}$ Basically the immune system selects for infectious agents or in this case, tumor cells that can no longer be targeted. Immunoediting has been shown for several cells types including the P1A tumor model where immunoediting of a key CTL epitope has been demonstrated in vitro after extensive culture with a TCR transgenic $\mathrm{CD} 8^{+} \mathrm{T}$ cell clone. ${ }^{10}$ Spreading and possible immunoediting has also been shown in patients with earlystage breast cancer and after dendritic cell-based immunotherapy for the treatment of malignant melanoma. ${ }^{34,35}$ Some tumors may be able to co-opt interactions with surrounding macrophages in the microenvironment to increase immune evasion. ${ }^{32}$ It is not surprising that immune effectors sculpt tumors for aggressiveness and immune subversion, but $\mathrm{T}$ cells may not be the only contributors to this selection. It has been shown that neutrophils may also immunosculpt tumors for such behavior. ${ }^{33}$

The advantage of using immunotherapy to target tumor vasculature is that it can be combined with other therapies, such as biologics, that directly target the tumor. In addition, using a therapeutic vaccine also provides the additional advantage of being able to boost the response at a later time. However, one of the major drawbacks of targeting tumors using immunotherapy is that the patient must be immunocompetent for vaccination. Chemotherapeutics usually involve the use of antiproliferative and/or antimetabolic drugs such as cyclophosphamide and methotrexate, respectively. Used at very high concentrations this usually results in immune ablation and/or susceptibility to new infections. In addition, immunotherapy using a live bacteria vector poses additional problems for the now immunocompromised patient. This is why several attempts have been made to further attenuate these Lm-vectors for human use. ${ }^{36}$ Immunotherapy is best implemented as a second-line therapy, in an adjuvant setting, post-surgery, and in combination with an antibody biologic if possible. The advantage of immunotherapy is greatest when fighting recurring disease or metastasis. Targeting newly forming vessels within micrometastases is where Flk-1 vaccines have the greatest potential. Epitope spreading to a tumor-associated antigen, such as Her-2/neu, is a secondary step, but does appear to be necessary for tumor control..$^{13}$ Immunological escape through immunoediting of tumor-associated antigens demands the need for combined immunotherapy where both the vasculature and tumor-associated antigens can be targeted simultaneously. A chimera or combinatorial vaccine fusing together the key epitopes of Flk-1 and Her-2/neu would be the best option for fighting immunological escape as demonstrated in this report. ${ }^{5}$ Sequential vaccination or mixed vaccines could substitute as a second option.

Here we show that vaccination against the vasculature can induce epitope spreading to Her-2/neu and delay tumor onset in a spontaneous model for Her- $2 /$ neu $^{+}$breast cancer. However, tumors can still escape immunological recognition via mutation of key residues, which may be responsible for either CTL recognition or anchoring to MHC-1 molecules, in endogenous tumor antigens that are not the target of the vaccine. Further investigation is warranted to combine multiple vaccination strategies or treatment strategies to increase tumor surveillance while simultaneously inhibiting new cancers. Combined or sequential immunization with mismatched vaccines may confer additional therapy and outpace the ability of the tumor to escape by immunoediting.

\section{Disclosure}

Grant support was received from American Association of Cancer Research Translational Breast Cancer Grant (AACRBCRF) and RO1CA109253 (Yvonne Paterson). Matthew Seavey was supported by training grant "The immunobiology of normal and neoplastic lymphocytes" T32CA09140, during the time in which this work was being conducted.

Yvonne Paterson has a financial interest in Advaxis, Inc., a vaccine and therapeutic company that has licensed or has an option to license all patents from the University of Pennsylvania that concern the use of Listeria monocytogenes or listerial products as vaccines.

\section{Acknowledgments}

We thank Dr Geetha Muthukumaran for reviewing this manuscript. We would like to acknowledge Drs Paulo Maciag, Nada Al-Rawi, and Duane Sewell for help with the production and generation of the Lm-LLO-Flk-1 vaccines. We would also like to thank Dr Reshma Singh for her early work in setting-up the Her-2/neu breast cancer mouse model system and for initially documenting the Her-2/neu mutation phenomenon. In addition, we would like to thank the Children's Hospital of Philadelphia and University of Pennsylvania sequencing cores for help and assistance.

\section{References}

1. Harzstark AL, Small EJ. Immunotherapy for prostate cancer using antigen-loaded antigen-presenting cells: APC8015 (Provenge). Expert Opin Biol Ther. 2007; 7:1275-1280.

2. Rosenberg SA, Restifo NP, Yang JC, Morgan RA, Dudley ME. Adoptive cell transfer: a clinical path to effective cancer immunotherapy. Nat Rev Cancer. 2008;8:299-308.

3. Wood LM, Guirnalda PD, Seavey MM, Paterson Y. Cancer immunotherapy using Listeria monocytogenes and listerial virulence factors. Immunol Res. 2008;42:233-245. 
4. Hussain SF, Paterson Y. What is needed for effective antitumor immunotherapy? Lessons learned using Listeria monocytogenes as a live vector for HPV-associated tumors. Cancer Immunol Immunother. 2005;54:577-586.

5. Seavey MM, Pan ZK, Maciag PC, et al. A novel human Her-2/neu chimeric molecule expressed by Listeria monocytogenes can elicit potent HLA-A2 restricted CD8-positive T cell responses and impact the growth and spread of Her-2/neu-positive breast tumors. Clin Cancer Res. 2009;15:924-932.

6. Shahabi V, Reyes-Reyes M, Wallecha A, Rivera S, Paterson Y, Maciag PC. Development of a Listeria monocytogenes based vaccine against prostate cancer. Cancer Immunol Immunother. 2008;57: 1301-1313.

7. Singh R, Dominiecki ME, Jaffee EM, Paterson Y. Fusion to Listeriolysin O and delivery by Listeria monocytogenes enhances the immunogenicity of HER-2/neu and reveals subdominant epitopes in the FVB/N mouse. J Immunol. 2005;175:3663-3673.

8. Singh R, Paterson Y. Vaccination strategy determines the emergence and dominance of $\mathrm{CD}^{+} \mathrm{T}$-cell epitopes in a $\mathrm{FVB} / \mathrm{N}$ rat HER-2/neu mouse model of breast cancer. Cancer Res. 2006;66:7748-7757.

9. Souders NC, Sewell DA, Pan ZK, et al. Listeria-based vaccines can overcome tolerance by expanding low avidity $\mathrm{CD}^{+} \mathrm{T}$ cells capable of eradicating a solid tumor in a transgenic mouse model of cancer. Cancer Immun. 2007;7:2.

10. Bai XF, Liu J, Li O, Zheng P, Liu Y. Antigenic drift as a mechanism for tumor evasion of destruction by cytolytic T lymphocytes. J Clin Invest. 2003;111:1487-1496.

11. Singh R, Paterson Y. Immunoediting sculpts tumor epitopes during immunotherapy. Cancer Res. 2007;67:1887-1892.

12. Pothlichet J, Heidmann T, Mangeney M. A recombinant endogenous retrovirus amplified in a mouse neuroblastoma is involved in tumor growth in vivo. Int J Cancer Suppl. 2006;119:815-822.

13. Seavey MM, Maciag PC, Al-Rawi N, Sewell D, Paterson Y. An antivascular endothelial growth factor receptor 2/fetal liver kinase-1 Listeria monocytogenes anti-angiogenesis cancer vaccine for the treatment of primary and metastatic Her- $2 /$ neu $^{+}$breast tumors in a mouse model. J Immunol. 2009;182:5537-5546.

14. Park DI, Kang MS, Oh SJ, et al. HER-2/neu overexpression is an independent prognostic factor in colorectal cancer. Int $J$ Colorectal Dis. 2007;22:491-497.

15. Sanna G, Franceschelli L, Rotmensz N, et al. Brain metastases in patients with advanced breast cancer. Anticancer Res. 2007;27:2865-2869.

16. Gaedcke J, Traub F, Milde S, et al. Predominance of the basal type and HER-2/neu type in brain metastasis from breast cancer. Mod Pathol. 2007;20:864-870.

17. Mata M, Yao ZJ, Zubair A, Syres K, Paterson Y. Evaluation of a recombinant Listeria monocytogenes expressing an HIV protein that protects mice against viral challenge. Vaccine. 2001;19:1435-1445.

18. Luo Y, Markowitz D, Xiang R, Zhou H, Reisfeld RA. Flk-1-based minigene vaccines induce $T$ cell-mediated suppression of angiogenesis and tumor protective immunity in syngeneic BALB/c mice. Vaccine. 2007;25:1409-1415.

19. Maciag PC, Seavey MM, Pan ZK, Ferrone S, Paterson Y. Cancer immunotherapy targeting the high molecular weight melanoma-associated antigen protein results in a broad antitumor response and reduction of pericytes in the tumor vasculature. Cancer Res. 2008;68:8066-8075.
20. Peng X, Treml J, Paterson Y. Adjuvant properties of listeriolysin O protein in a DNA vaccination strategy. Cancer Immunol Immunother. 2007;56:797-806.

21. Shahabi V, Reyes-Reyes M, Wallecha A, Rivera S, Paterson Y, Maciag PC. Development of a Listeria monocytogenes based vaccine against prostate cancer. Cancer Immunol Immunother. 2008;57:1301-1313.

22. Schlegel J, Trenkle T, Stumm G, Kiessling M. Growth inhibition by dominant-negative mutations of the neu-encoded oncoprotein. Int $J$ Cancer Suppl. 1997;70:78-83.

23. Burgess AW. EGFR family: structure physiology signalling and therapeutic targets. Growth Factors. 2008;26:263-274.

24. Singh R, Paterson Y. In the FVB/N HER-2/neu transgenic mouse both peripheral and central tolerance limit the immune response targeting HER-2/neu induced by Listeria monocytogenes-based vaccines. Cancer Immunol Immunother. 2007;56:927-938.

25. Huntzicker EG, Estay IS, Zhen H, Lokteva LA, Jackson PK, Oro AE. Dual degradation signals control Gli protein stability and tumor formation. Genes Dev. 2006;20:276-281.

26. Yue S, Chen Y, Cheng SY. Hedgehog signaling promotes the degradation of tumor suppressor Sufu through the ubiquitin-proteasome pathway. Oncogene. 2009;28:492-499.

27. Chang CC, Campoli M, Restifo NP, Wang X, Ferrone S. Immune selection of hot-spot beta 2-microglobulin gene mutations, HLA-A2 allospecificity loss, and antigen-processing machinery component down-regulation in melanoma cells derived from recurrent metastases following immunotherapy. J Immunol. 2005;174:1462-1471.

28. Quesnel A, Hsu SC, Delmas A, Steward MW, Trudelle Y, Abastado JP. Efficient binding to the MHC class I K(d) molecule of synthetic peptides in which the anchoring position 2 does not fit the consensus motif. FEBS Lett. 1996;387:42-46.

29. Tian J, Gregori S, Adorini L, Kaufman DL. The frequency of high avidity T cells determines the hierarchy of determinant spreading. J Immunol. 2001;166:7144-7150.

30. Chen J, Deng YM. Influenza viral antigen variation, host antibody production and new approach to control epidemics. Virol J. 2009;6:30.

31. Pham VP, Rothman PB, Gottesdiener KM. Binding of trans-acting factors to the double-stranded variant surface glycoprotein (VSG) expression site promoter of Trypanosoma brucei. Mol Biochem Parasitol. 1997;89:11-23.

32. Pukrop T, Klemm F, Hagemann T, et al. Wnt 5 a signaling is critical for macrophage-induced invasion of breast cancer cell lines. Proc Natl Acad Sci US A. 2006;103:5454-5459.

33. De Larco JE, Wuertz BR, Furcht LT. The potential role of neutrophils in promoting the metastatic phenotype of tumors releasing interleukin-8. Clin Cancer Res. 2004;10:4895-4900.

34. Mittendorf EA, Gurney JM, Storrer CE, Shriver CD, Ponniah S, Peoples GE. Vaccination with a HER-2/neu peptide induces intra- and inter-antigenic epitope spreading in patients with early stage breast cancer. Surgery. 2006;139(3):407-418.

35. Butterfield LH, Ribas A, Dissette VB, et al. Determinant spreading associated with clinical response in dendritic cell-based immunotherapy for malignant melanoma. Clin Cancer Res. 2004;9(3):998-1008.

36. Wallecha A, Maciag PC, Rivera S, Paterson Y, Shahabi V. Construction and characterization of an attenuated Listeria monocytogenes strain for clinical use in cancer immunotherapy. Clin Vaccine Immunol. 2009;16(1):96-103.

\section{Publish your work in this journal}

Breast Cancer: Targets and Therapy is an international, peerreviewed open access journal focusing on breast cancer research, identification of therapeutic targets and the optimal use of preventative and integrated treatment interventions to achieve improved outcomes, enhanced survival and quality of life for the cancer

\section{Dovepress}

patient. View the full aims and scopes of this journal here. The manuscript management system is completely online and includes a very quick and fair peer-review system, which is all easy to use. Visit http://www.dovepress.com/testimonials.php to read real quotes from published authors. 